\title{
Parylene-Based Electrochemical-MEMS Transducers
}

\author{
Christian A. Gutierrez, Member, IEEE, and Ellis Meng, Senior Member, IEEE
}

\begin{abstract}
We report the design, fabrication, and characterization of electrochemical microelectromechanical systems (EC-MEMS) devices featuring encapsulated fluid as the basis for transduction. Parylene microstructures, including discrete chambers (square or circular geometry), are utilized as physical transducers for electrochemically mediated liquid impedance transduction of physical phenomenon such as contact and force. Parylene-based EC-MEMS technologies uniquely leverage advantages in size $(<500 \mu \mathrm{m}$ diameter), packaging (no hermetic packaging necessary), power (nanowatts to microwatts), and flexibility to address the physical sensing requirements of in vivo applications. Robust EC impedance (EI) sensor responses (up to $20 \%$ from base-line) and discrimination of $200-\mathrm{nm}$ chamber deflections were possible using the EI transduction technique. Additional transducer configurations enabling electrolysis-based out-of-plane actuation and biomimetic mechanotransduction in microfluidic channels are also presented.

[2010-0157]
\end{abstract}

Index Terms-Biomimetic, contact sensor, electrochemical microelectromechanical systems (EC-MEMS), EC sensing, EC transduction, electrolysis actuation, force sensor, impedance-based sensor, liquid encapsulation, liquid impedance, mechanotransduction, Parylene C.

\section{INTRODUCTION}

$\mathbf{E}$ LECTROCHEMICAL (EC) transducer technology is well established and used in a variety of applications. Electrochemically based sensing, for example, is broadly applied to fields ranging from analytical chemistry to macromolecular and cellular detection and analysis [1]. It was extensively applied in capillary electrophoresis-based systems for macromolecular characterization of deoxyribonucleic acid and neurotransmitters [2]-[4] and gained popularity as a microfluidic-based sensing and actuation scheme for lab-on-a-chip and micro total analysis systems technologies [1], [5]-[7]. EC sensing was perhaps most famously utilized in the invention of the Coulter counter, by Wallace H. Coulter, in the late 1940s [8], which

Manuscript received May 28, 2010; revised July 14, 2010; accepted August 5, 2010. Date of publication October 11, 2010; date of current version November 30, 2010. This work was supported in part by the Engineering Research Centers Program of the National Science Foundation (NSF) under Award EEC-0310723, in part by an NSF CAREER Award (ECS-0547544), and in part by the Bill and Melinda Gates Foundation (CG). Subject Editor C.-J. Kim.

C. A. Gutierrez is with the Department of Biomedical Engineering and the Biomimetic Microelectronic Systems Engineering Research Center, University of Southern California, Los Angeles, CA 90089 USA (e-mail: cagutier@usc.edu).

E. Meng is with the Department of Biomedical Engineering, University of Southern California, Los Angeles, CA 90089 USA, and also with the Ming Hsieh Department of Electrical Engineering and also with the Biomimetic Microelectronic Systems Engineering Research Center, University of Southern California, Los Angeles, CA 90089 USA (e-mail: ellis.meng@usc.edu).

Color versions of one or more of the figures in this paper are available online at http://ieeexplore.iee.org.

Digital Object Identifier 10.1109/JMEMS.2010.2076791 utilizes the EC Coulter principle for particle detection and analysis.

Despite the widespread use in such biological and chemical analysis systems, EC sensing technology has seen little application in other modes of transduction particularly that of physical quantities such as contact detection, force, and pressure. The inherent simplicity and sensitivity of EC transduction lend itself well to physical transduction applications. Monitoring fluid response to an external physical stimulus via EC impedance (EI) requires only the interfacing of electrodes with the electrolyte fluid and the application of low-power electrical measurement signals; both are easily realized in standard microelectromechanical systems (MEMS) devices. However, to date, EI-based sensing has been exploited only in limited applications. Large-format EI-based tactile sensors for robotics have demonstrated detection of multiple parameters, including point contact, force, direction, object shape, and texture [9][11], and bubble-based hydrostatic pressure transduction was shown within microfluidic channels using EI-based transduction [12]. EC-based physical transduction is an emerging field ideally suited to the unique tools and capabilities of MEMS fabrication techniques. EC-MEMS technology leverages the ability to precisely pattern and define features (both electrical and mechanical) at the micrometer scale and the proven capability of microfluidic technology to realize novel transducer modalities.

Polymer-based EC-MEMS is of particular interest for its potential application to in vivo settings where sensing of physical parameters such as contact, force, pressure, and strain must occur in a compliant low-modulus wet environment. These conditions are particularly difficult for standard transduction techniques, such as piezoresistive or capacitive, which generally cannot operate in wet conditions and often require hermetic packaging. Quantification of parameters such as contact force and pressure is of particular importance for neuromodulation technologies such as epiretinal electrode arrays for retinal prostheses that intimately contact nervous tissues at scales below $500 \times 500 \mu \mathrm{m}^{2}$ [13], [14]. Excess mechanical loading of sensitive tissues, such as retina, can lead to adverse effects, including mechanical insult and apoptotic cell death [15]-[17]. Thus, understanding the extent of this acute and chronic contact is critical to maintaining tissue viability, device efficacy, and overall functionality. However, the magnitude of this mechanical interaction has not been quantitatively studied and is currently unknown. There is evidence that suggests that micronewton-to-millinewton forces can be expected [18]-[21]; however, measurement of these interfacial forces in situ requires novel sensor technology. Polymer-MEMS sensors based on EI transduction offer a novel approach with many advantages, including improved mechanical matching to tissue (in 


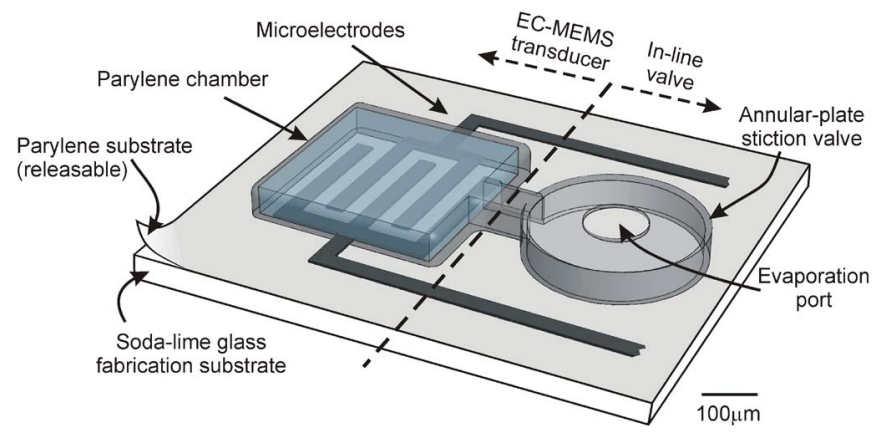

Fig. 1. Model of Parylene-based EC-MEMS device. An electrolyte-filled microchamber forms the core of the transducer, and an in-line stiction valve provides liquid encapsulation capability. The stiction valve, as depicted, is open. (Device layout adapted from [23].)

contrast to rigid materials such as silicon and glass), small footprint, low power consumption, simple construction, and excellent sensitivity [22].

In this paper,we present the development of a Parylene-based EC-MEMS device utilizing encapsulated fluid to mediate transduction designed specifically for the measurement of interfacial contact forces between implants and tissue. The key elements of the device design are an electrolyte-filled Parylene chamber which provides a soft compliant contact surface and a minimum of two microelectrodes exposed to the fluidic contents of the structure (Fig. 1). The exposed electrodes serve as the electrical interface for EI measurements. To ensure liquid entrapment within the chamber structure, an in-line annular-plate stictionbased valve is utilized. The stiction valve design and concept were previously described by Zhang et al. [23]. Here, their application to EI-based contact sensing at scales below $500 \times$ $500 \mu \mathrm{m}^{2}$ using Parylene microchamber structures is presented and discussed. Additional EC-MEMS applications related to microactuation and fluid-enabled mechanotransduction are also presented.

\section{BACKGRound AND THEORETICAL CONSIDERATIONS}

When a voltage is applied to an electrode in contact with an electrolyte, the electrode interacts capacitively with the electrolyte, generating ionic current flow by non-Faradaic mechanisms [24]. John E. B. Randles, one of the most important theorists in the field of electrochemistry, devised a circuit model in 1947 to describe the electrode-electrolyte junction [25]. He described the interface as a capacitor, the Helmholtz double-layer capacitance $C_{\mathrm{dl}}$, in parallel with a resistance to charge transfer $R_{\mathrm{ct}}$. This parallel combination describes the electrode-electrolyte interface and is in series with a solution resistance $R_{S}$ [see (1)].

By applying an alternating current, the impedance of the electrolyte volume around the electrodes can be measured. In our device structure, the electrolyte volume is confined near the electrodes by a polymer microchamber. Thus, externally applied forces to the microchamber result in volumetric deformation of the electrolyte that induces corresponding volumetric variations to the fluid path of current-carrying ions between the electrodes. The resulting impedance changes contain information about the magnitude of the externally applied force (Fig. 2).
At sufficiently high frequencies $(>1 \mathrm{kHz}), C_{\mathrm{dl}}$ essentially acts as a short, and the solution resistance $R_{S}$ dominates the impedance response. It is generally desirable for the fluid to have a high resistivity to ensure that the measured impedance of the series circuit (electrode leads plus fluid) is dominated by $R_{S}$. Consequently, the impedance response can be approximately modeled as a simple classical resistance, where $\rho$ is the resistivity of the electrolyte, $l$ is the distance between the electrodes, and $A$ is the cross-sectional area of the fluid between the electrodes [see (2)]. The impedance is therefore inversely proportional to the cross-sectional area between the electrodes and is directly analogous to a variable resistor. This phenomenon enables the transduction and measurement of physical quantities such as contact force and contact pressure acting on the electrolyte-filled chamber

$$
\begin{aligned}
Z & =\frac{1}{\frac{1}{R_{\mathrm{ct}}}+j w C_{\mathrm{dl}}}+R_{S} \\
|Z|_{f>1 \mathrm{khz}} & \approx R_{S}=\frac{\rho l}{A} .
\end{aligned}
$$

It is desirable to apply an excitation signal to the electrode system in such a way that the voltages developed across the metal-electrolyte interfaces are sufficiently low to avoid nonreversible Faradaic reactions, which could potentially corrode the metal contacts and cause electrolysis of the electrolytic fluid [26]. This is accomplished by applying a small alternating current (typically $<1 \mu \mathrm{A}$ ) and measuring the resulting voltage across the electrodes. As a result, this measurement is low power and typically requires no more than a few microwatts. At these low powers, no significant increase in temperature is observed as most of the energy is expended by reversible isothermal Faradaic reactions at the electrode surface, resulting in negligible ohmic losses.

\section{Device Design}

Device architecture consists of the following: 1) an electrolyte-filled Parylene chamber that provides a soft compliant contact surface and 2) interdigitated electrodes exposed to the fluidic contents trapped in the chamber that serve as the electrical interface for EC measurements (Fig. 3). Microchamber structures of square and circular geometries were fabricated. The primary design goal was to enable the controlled entrapment of small liquid volumes on the order of $\sim 1 \mathrm{~nL}$ for physical EC transducers. A polymer structural material was favored because of the material compliance enabling the fabrication of an easily deformable structure at scales below $500 \times 500 \mu \mathrm{m}^{2}$. Furthermore, the development of such technologies for implantable or in vivo applications warrants the use of flexible biocompatible materials that are amenable to standard microfabrication techniques. These design goals were addressed by using Parylene C (United States Pharmacopoeia Class VI biocompatibility) as the structural material in the construction of our devices [27], [28]. Additionally, the ability to fabricate these devices completely out of Parylene $\mathrm{C}$ enables the release from rigid substrates and provides the ability to place these sensors on nonplanar surfaces (such as tissue) and 


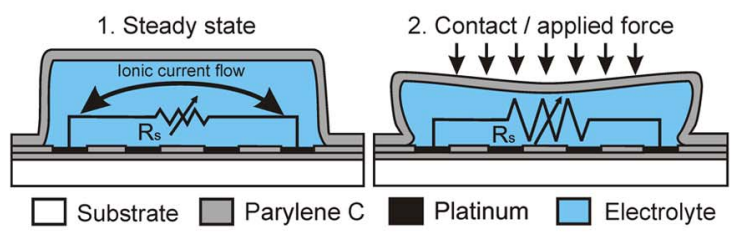

(a)

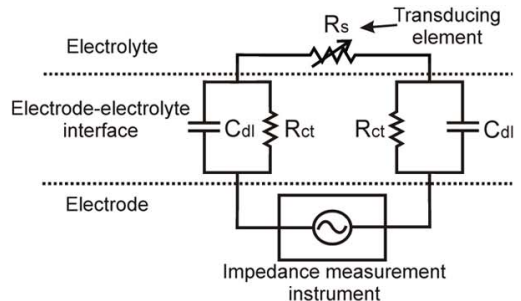

(b)

Fig. 2. Depiction of EI sensing principle using an encapsulated liquid volume. (a) Under applied forces, the cross-sectional area between the electrodes decreases, resulting in an increase in $R_{S}$ and, thus, impedance magnitude. (b) Randles equivalent circuit model, where $C_{\mathrm{dl}}$ is the electrode double-layer capacitance, $R_{S}$ is the solution resistance, and $R_{\mathrm{ct}}$ is the charge transfer resistance.

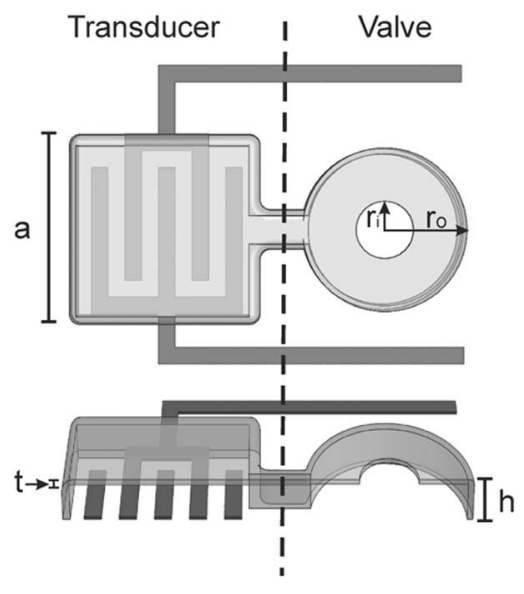

Fig. 3. Key dimensional design parameters for transducer and annular plate stiction valve. Dimension $a$ represents the side length for square chambers and diameter for circular transducer chambers.

in other locations and environments which are very difficult to access with traditional materials.

To minimize footprint and provide automatic liquid encapsulation capability to each device, an in-line Parylene-based stiction valve was integrated next to the sensing chamber. Due to the liquid-mediated device functionality and the desire to avoid tedious encapsulation techniques such as epoxy or sealants, a self-sealing approach was adopted through the use of a stiction valve. Parylene-based stiction valves were successfully demonstrated for long-term liquid encapsulation and employ simple design guidelines and straightforward fabrication techniques [23], [29]. Integration of the automatic fluid sealing mechanism in line with the devices also enables batch sealing of multiple devices on a wafer, providing significant packaging advantages over traditional serial fluid sealing techniques.

\section{A. Stiction-Mediated Liquid Encapsulation}

Stiction is usually considered a notorious and unwanted failure mechanism in MEMS and can render devices inoperable unless accounted for appropriately [30], [31]. This phenomenon, however, is beneficial when utilized as a valve sealing mechanism. The analysis and design guidelines for annularplate stiction valves were previously reported in [23] and [32] and adopted here. Briefly, stiction of a suspended Parylene annular plate occurs for critical number $N_{c}>1$, where

$$
N_{c}=\frac{\sigma r_{o}^{4}}{D h^{2}} p(\phi, v)
$$

TABLE I

DeVice Dimensions

\begin{tabular}{c|c}
\hline $\begin{array}{l}\text { Geometric } \\
\text { parameter }\end{array}$ & Dimension $(\boldsymbol{\mu m})$ \\
\hline$h$ & $10-22$ \\
\hline$t$ & $2-8$ \\
\hline$a$ & $250,300,350,400,500$ \\
\hline$r_{o}$ & 165 \\
\hline$r_{i}$ & 50 \\
\hline
\end{tabular}

with surface tension term $\sigma=2 \gamma_{\mathrm{LA}} \cos \theta_{C}$ and flexural rigidity $D=\left(E t^{3} / 12\left(1-v^{2}\right)\right)$. Here, $\gamma_{\mathrm{LA}}$ is the liquid-air surface tension, $\theta_{C}$ is the Parylene contact angle, $E$ is Young's Modulus, $t$ is the membrane thickness, $h$ is the membrane height, $v$ is Poisson's ratio, $\phi$ is the ratio $r_{i} / r_{o}$, and $p$ is a numerical function of $\phi$ and $v$. To ensure liquid entrapment within the chamber, stiction valve dimensions were selected to ensure that the condition $N_{c} \gg 1$ was satisfied (Table I).

\section{Device Fabrication}

The devices were fabricated utilizing standard surface micromachining techniques on a 3-in soda-lime wafer (see Figs. 4 and 5). The entire process was achieved at relatively low temperatures (below $100{ }^{\circ} \mathrm{C}$ ), which is compatible with a variety of polymers. The wafer was optionally treated with Silane A-174 Parylene adhesion promoter (Specialty Coating Systems, Inc., Indianapolis, ID). Inclusion of the adhesion promoter ensures that the underlying Parylene substrate, upon which the devices are surface micromachined, adheres to the soda-lime glass supporting substrate during wafer dicing. A 10- $\mu \mathrm{m}$ Parylene $\mathrm{C}$ film was then vapor deposited on the entire wafer. A 2000- $\AA$ layer of platinum was deposited by e-beam evaporation and patterned by a liftoff process briefly described here. A dual-layer photoresist process was used to create an undercut sidewall profile to facilitate metal liftoff. First, AZ1518 photoresist (AZ Electronic Materials, Branchburg, NJ) was spun at $4000 \mathrm{r} / \mathrm{min}$ followed by global exposure. Then, AZ4400 photoresist (AZ Electronic Materials, Branchburg, NJ) was applied at $4000 \mathrm{r} / \mathrm{min}$ and patterned. Following a short descum in oxygen plasma, the Pt film was e-beam evaporated and patterned, forming the chamber electrodes, leads, and contact pads. A sacrificial photoresist layer $(h=10-22 \mu \mathrm{m})$ of AZ4620 (AZ Electronic Materials, Branchburg, NJ) was spun and patterned to define the chamber and valve structures $\left(r_{o}=165 \mu \mathrm{m}\right)$. A second layer of Parylene $(t=2-8 \mu \mathrm{m})$ was 


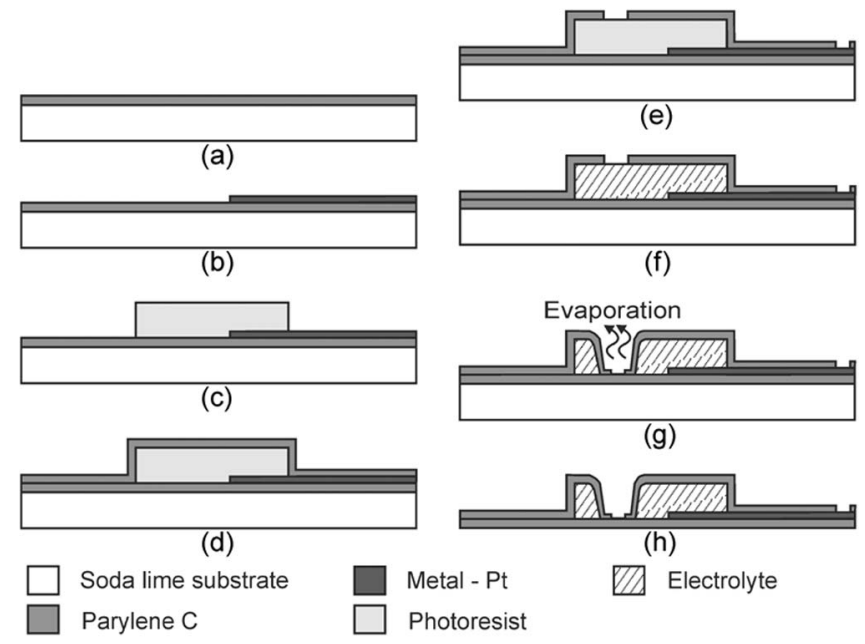

Fig. 4. Fabrication process for EC-MEMS Parylene-based contact sensor. (a) Parylene deposition. (b) Deposit and pattern electrodes. (c) Pattern sacrificial photoresist. (d) Deposit Parylene. (e) Etch Parylene over valve. (f) Dissolve sacrificial photoresist and replace with electrolyte. (g) Initiate evaporative stiction valve closure. (h) Release from substrate.

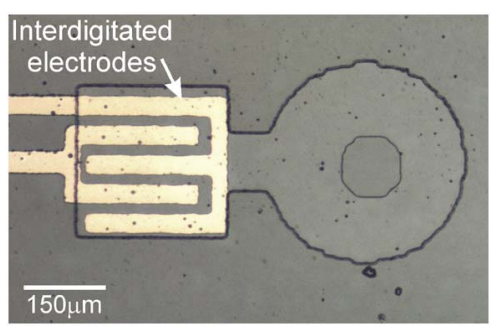

Fig. 5. Fabricated Parylene microchamber structure with in-line stiction valve. Interdigitated platinum electrodes are integrated and exposed to the chamber liquid environment.

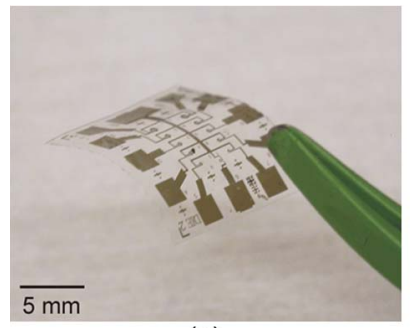

(a)

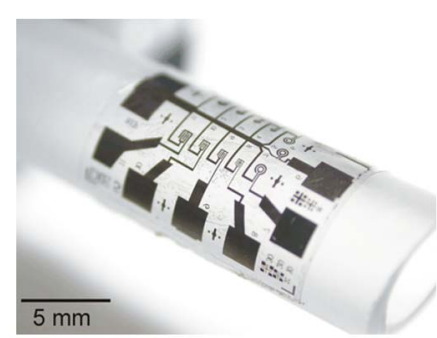

(b)
Fig. 6. (a) Released Parylene film with integrated EC-MEMS devices. (b) Released device film adhered to curved surface.

then deposited. The Parylene over the contact pads and valve orifice $\left(r_{i}=50 \mu \mathrm{m}\right)$ was then etched in oxygen plasma (100 W and $100 \mathrm{mT}$ ) using a photoresist etch mask (AZ 4620). Due to the comparable etch rates (approximately 1:1) of Parylene and photoresist, the thickness of the etch mask was at least 1.5 times the thickness of the Parylene film being etched. After etching, the remainder of the photoresist etch mask was left on the surface to serve as a protective layer during dicing. Square test dies $\left(1 \mathrm{~cm}^{2}\right)$, each containing several test devices, were diced for characterization and testing. For wafers untreated with A-174, devices were released at this stage by cutting the Parylene film with a razor blade and peeling from the substrate (Fig. 6).
To prepare the devices for use, it was necessary to remove the sacrificial photoresist contained within the Parylene transducer chamber and fill the device with the desired electrolyte. This was accomplished by first immersing the entire die in an acetone bath for approximately $30 \mathrm{~min}$. The acetone dissolved the protective photoresist layer left on the surface from the prior dicing step as well as the sacrificial photoresist within the Parylene chamber which escaped through the valve orifice opened in the final Parylene etching step. Following immersion in acetone, the devices were immediately transferred to an isopropyl alcohol bath to rinse away the acetone and dissolve the photoresist $(\sim 15 \mathrm{~min})$. The devices were then transferred to a deionized (DI) water bath to rinse away any remaining solvents. Following the rinse, the devices were placed in a bath of the desired electrolyte for about $30 \mathrm{~min}$ to ensure complete filling. The entire sacrificial photoresist removal and filling process is diffusion driven, so adequate time is required for each complete solution replacement step within the Parylene microchamber. Devices were then removed from the final bath and exposed to the ambient laboratory environment $\left(\sim 25{ }^{\circ} \mathrm{C}\right.$ and $35 \%$ relative humidity) for drying. Within minutes, evaporation through the valve orifice created the necessary conditions for the stictioninduced collapse of the annular valve plate to the substrate, thereby encapsulating the remaining liquid in the chamber.

\section{EXPERIMENTAL METHODS}

\section{A. Impedance Measurement Interface}

Impedance measurements were made with a high-precision impedance converter board (AD5933 Analog Devices, Norwood, MA). The AD5933 combines an onboard frequency generator with a 12-bit $10^{6}$ sample-per-second analog-to-digital converter (ADC). The frequency generator allows an external complex impedance to be excited at a known frequency. The impedance response signal was sampled by the onboard ADC, and a discrete Fourier transform was obtained using onboard signal processing. The converter board was interfaced via USB to a PC where a custom LabVIEW interface (National Instruments, Austin, TX) was developed for real-time data acquisition. An ac drive signal of $1 \mathrm{~V}_{\mathrm{pp}}$ at $5 \mathrm{kHz}$ was selected to avoid any irreversible Faradaic reactions at the electrode surface. At this frequency, the double-layer capacitance was effectively shorted, bypassing the charge transfer resistance, which allowed the solution resistance to dominate the measured impedance.

\section{B. Experimental Setup}

Characterization experiments were conducted on a $1-\mathrm{cm}^{2}$ test die mounted on a glass slide. A drop of mineral oil was used to minimize evaporative water loss through the Parylene. DI water was selected as the electrolyte because of the inherently high solution resistance. Due to trace impurities and dissolved gases, such as carbon dioxide, from the ambient environment, DI water exhibits a finite and measurable impedance. Impedance measurements were generally in the megaohms range at the measurement frequency. 


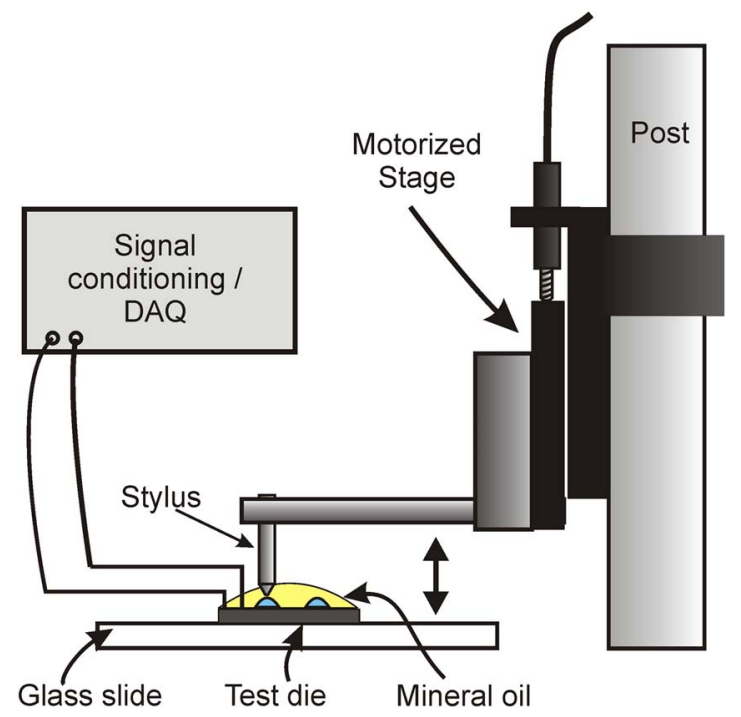

Fig. 7. Experimental setup.

The experimental setup was built on a floating optical table and consisted of a motorized micropositioning stage with submicrometer accuracy (Thorlabs Inc., Newton, NJ) to precisely control the indentation depth of an attached microprobe stylus tip (Fig. 7). The stylus tip had a diameter of approximately $100 \mu \mathrm{m}$. The motorized stage and impedance converter board were interfaced through a custom LabVIEW interface for realtime data acquisition.

\section{RESUlTS AND DISCUSSION}

\section{A. Liquid Encapsulation}

Stiction valve sealing and liquid encapsulation capability were evaluated by using a sample die containing several identical devices under optical observation. The devices were filled, as described previously, with DI water as the electrolyte. The die was then removed from the bath and exposed to ambient laboratory conditions to initiate fluid encapsulation. Successful stiction valve sealing was clearly evidenced by the appearance of a dark central region indicating conformal contact between the annular plate and substrate. Emergence of interference rings between the central region and the periphery (as the valve plate transitioned from being pinned to the substrate to freestanding near the anchored edges) indicated complete circumferential sealing (see Figs. 8 and 9). Encapsulation of water within Parylene membranes was previously demonstrated [23], [29], [33]. Although Parylene has found ubiquitous application as a protective encapsulant, it is not hermetic. The water vapor transmission rate (WVTR) through the Parylene material itself is the limiting factor to encapsulation lifetime. Furthermore, for Parylene films less than about $8 \mu \mathrm{m}$ in thickness, pin holes, pores, and defects can exist that contribute to gas transport permeation through the film, thereby limiting the moisture barrier performance [34].

The WVTR $\left(5.6 \times 10^{-12} \mathrm{~g} \cdot \mu \mathrm{m} / \mu \mathrm{m}^{2} \cdot \mathrm{h}\right)$ of Parylene films was previously reported in literature [35]. Although DI water was utilized as the electrolyte here, alternative solutions, such as ethylene glycol-based conductive fluids, may be used
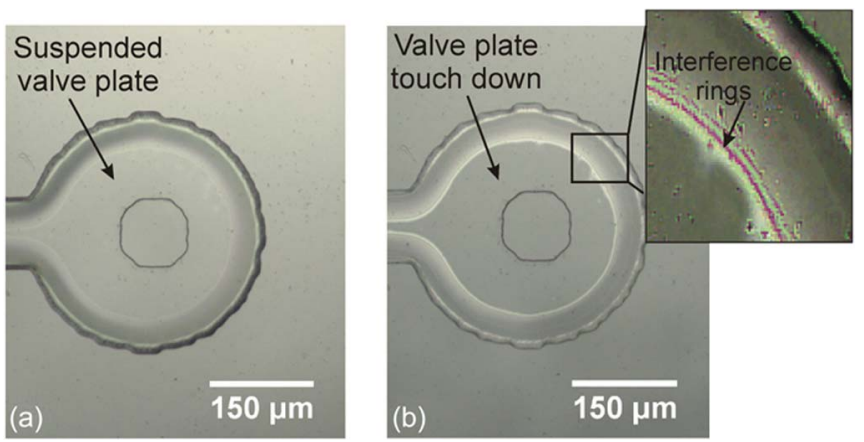

Fig. 8. (a) Open stiction valve immediately following removal from bath. (b) Closed valve. Interference rings (shown in contrast-enhanced inset image) indicate areas where the membrane transitions from collapse (center) to freestanding (periphery).
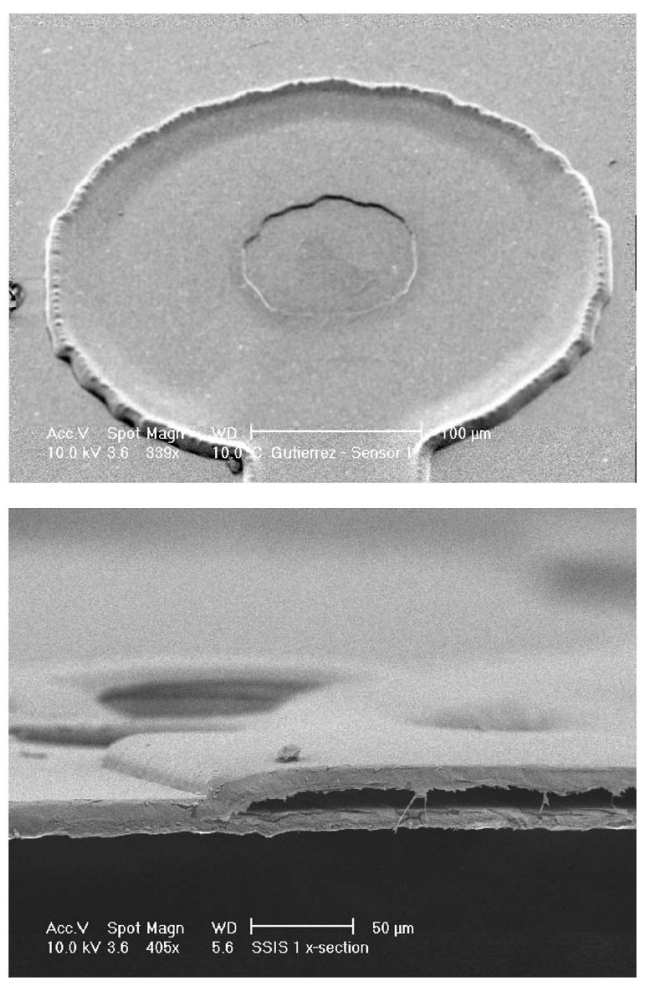

Fig. 9. (Top) SEM of collapsed stiction valve. (Bottom) SEM cross section taken through the transducer chamber.

for increased resistance to evaporation. For devices with $2-\mu$ m-thick Parylene membranes, the water vapor permeation through the Parylene film imposed a significant limitation to testing in the open-air laboratory environment. The volume of encapsulated liquid varied significantly over time, thereby introducing an unacceptable level of drift in addition to limiting total encapsulation time. Several methods exist to address this issue. Increasing the Parylene membrane thickness increases the encapsulation time proportionally, but a thicker membrane is also less compliant and results in a lower critical number $N_{c}$ required for stiction valve operation. Annealing of Parylene films $\left(200^{\circ} \mathrm{C}\right)$ was shown to reduce the WVTR by nearly $50 \%$ in addition to improving Parylene-Parylene adhesion [14], [35]. Additional coatings such as thin film metals have also improved barrier performance [23]. To mitigate evaporative 


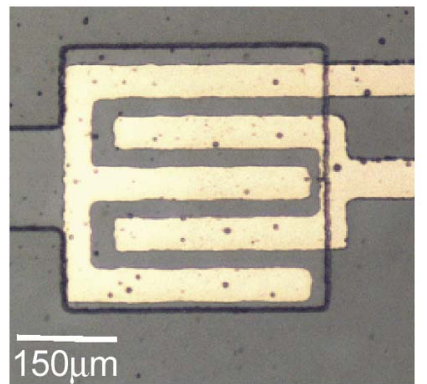

(a)

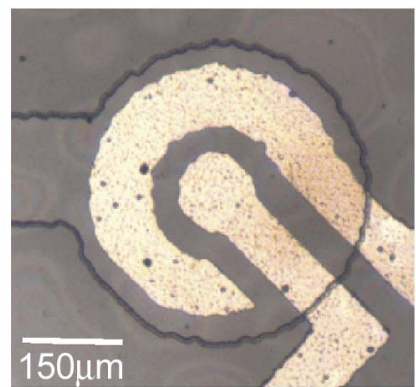

(b)
Fig. 10. (a) Square chamber with interdigitated electrodes. (b) Circular chamber with circumferential and central electrodes.
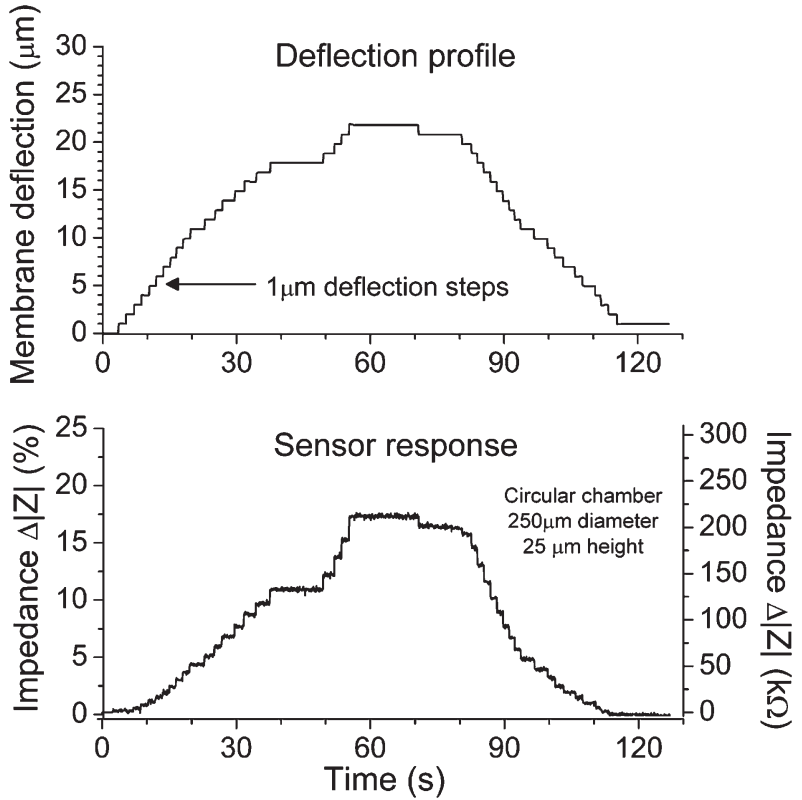

Fig. 11. (Top) Real-time deflection profile of the sensor membrane. Data show stepwise stage movement with attached stylus tip contacting sensor surface. (Bottom) Temporal response of sensor impedance to deflection. A $250-\mu \mathrm{m}-$ diameter sensor response is shown and is representative of typical sensor response.

effects, experiments were performed with a drop of mineral oil placed over the devices.

\section{B. Contact Sensing}

Devices were tested as physical contact sensors. Both square and circular chambers were fabricated. It was found that more robust responses were obtained with the circular chamber design. This may be in part due to the circularly symmetric electrode spacing in contrast to the serpentine electrode gap with the interdigitated design (Fig. 10). Applied deflections in such a design caused cross-sectional area changes along only a small region of the available electrode gap, resulting in small impedance changes $(<0.25 \%$ from baseline) that were difficult to detect using our measurement circuitry. Circular chambers of $250-, 300-$, and 500- $\mu \mathrm{m}$ diameters were tested. Each device had a chamber height of 22-25 $\mu \mathrm{m}$ and a membrane thickness of $8 \mu \mathrm{m}$. The sensors were deflected (and released) in 1- $\mu \mathrm{m}$ steps.

Temporal sensor response was robust and clearly tracked membrane deflection (Fig. 11). A clear sensor response was

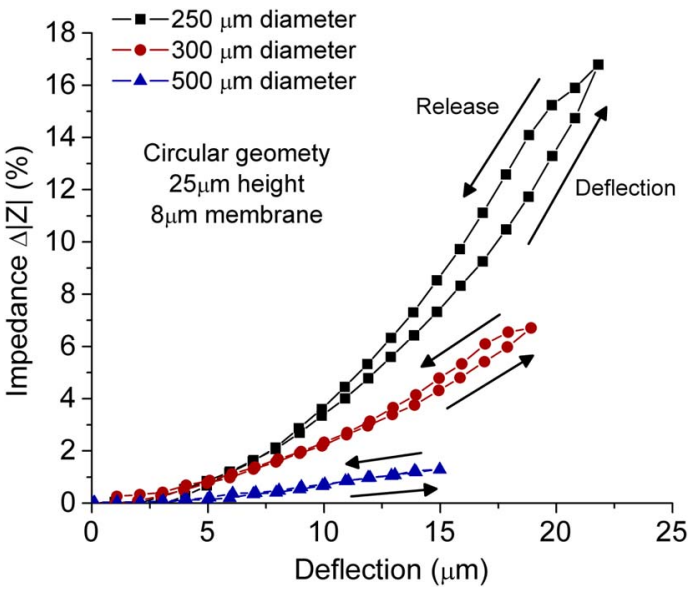

Fig. 12. Time-averaged impedance responses to $1-\mu \mathrm{m}$ deflection and release steps. Responses are shown for one full deflection and release cycle.

attained with deflections as small as $200 \mathrm{~nm}(0.8 \%$ resolution of full-scale deflection). In all experiments, deflection was limited to $22 \mu \mathrm{m}$ so as to not fully deform the chamber or achieve contact with the substrate which may scratch or otherwise damage the Parylene membrane. Impedance variations up to $20 \%$ from base line were achieved at maximum deflection, with $250-\mu \mathrm{m}$-diameter chambers demonstrating the large-signal response available with EI-based sensing. Sensor performance was consistent and reproducible for individual devices, but some variation was observed in the nominal resting (zerodeflection) impedance between devices. This variation was typically less than $10 \%$ and was attributed to the presence of a trace amount of residual photoresist particulates visible in the chamber electrolyte.

The impedance response to the first $10 \mu \mathrm{m}$ of deflection was less pronounced than the response near full deflection. Timeaveraged impedance responses clearly demonstrated this nonlinear behavior (Fig. 12). To further elucidate this relationship, cross-sectional area was calculated using theoretical deflection equations for rigidly clamped circular diaphragms [36]

$$
y=\frac{3\left(1-\mu^{2}\right) P}{16 E t^{3}}\left(a^{2}-r^{2}\right)^{2}
$$

where $y$ is the deflection, $P$ is the pressure, $t$ is the membrane thickness, $a$ is the diaphragm radius, $E$ is the modulus of elasticity, $r$ is the radial distance, and $\mu$ is Poisson's ratio.

The areas under the deflection curves were calculated and plotted against maximum deflection (Fig. 13). Cross-sectional area through the central axis of the diaphragm is at a minimum under the imposed deflection and is assumed to account for a majority of the resistive variations. Area clearly varies linearly with deflection, but the reciprocal dependence of impedance on the cross-sectional area dictates the observed nonlinear behavior [see (2)].

Furthermore, sensor sensitivity to deflection was found to vary with chamber size with smaller chambers exhibiting greater impedance-deflection sensitivity. This is in part due to proportionally larger fraction of the smaller chamber's surface area that is deflected by the stylus (fixed diameter), but generally smaller volumes (created by decreasing lateral footprint) 


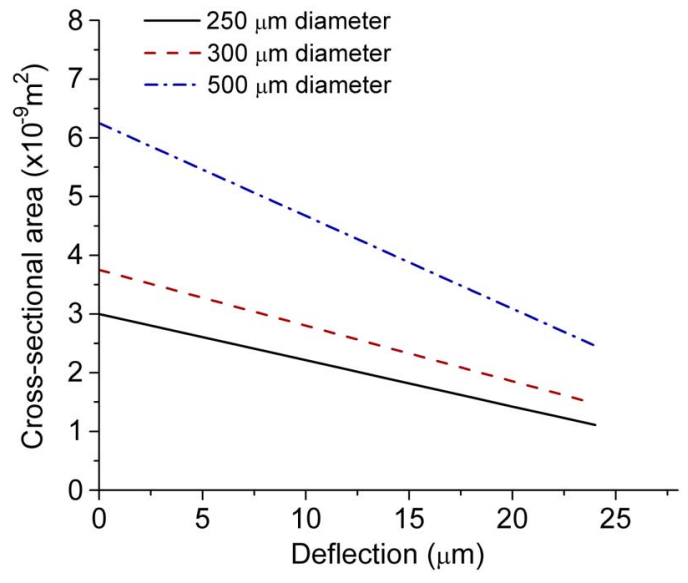

Fig. 13. Variation of theoretical cross-sectional area with deflection.

can be expected to be more sensitive to deflections for a given sensor height. The nonlinear sensor response is analogous to capacitive sensors, where plate separation is modulated and capacitance is inversely proportional to plate separation. Linearization techniques are available to transform calibrated nonlinear responses into a linear output. Once calibrated, linearization transformations can be easily implemented and programmed off-chip [37], [38].

The mechanical loads required to produce deflection of a (250- $\mu \mathrm{m}$-diameter and 8 - $\mu \mathrm{m}$-thick) chamber membrane were evaluated analytically and with finite-element modeling (FEM, COSMOSWorks 2009, Dassault Systèmes SolidWorks Corp, Concord, MA). Under a linear small-deflection approximation (deflection-to-thickness ratio < 0.3) [36] for a rigidly clamped circular diaphragm, a pressure of $1.27 \mathrm{MPa}(62.3-\mathrm{mN}$ total force) distributed over the sensor surface was required to produce a $20-\mu \mathrm{m}$ deflection. This value agreed well with the FEM result of $1.36 \mathrm{MPa}$. If a thin membrane under nonlinear large deflection is assumed (deflection-to-thickness ratio $>5$ ) [36], a uniform 4.5-MPa $(270-\mathrm{mN})$ pressure is required. The actual sensor response in the range of $20-\mu \mathrm{m}$ deflection (maximum deflection-to-thickness ratio $\approx 2.5$ ) is expected to be bounded by these two conditions. This response is sensitive to membrane thickness and radius, scaling with the thickness cubed and inversely with the radius to fourth power [see (4)]. Therefore, the membrane thickness and lateral chamber dimensions can be used to effectively tune the sensor response over a wide range of loading forces (micronewton to millinewton).

Impedance-based detection of surface deformations using a fluid-filled large-format $\left(1-\mathrm{cm}^{2}\right)$ sensor was previously described by Helsel et al. using a planar grid of gold-chromium electrodes patterned on a rigid $\mathrm{Al}_{2} \mathrm{O}_{3}$ substrate, ethylene glycol-based conductive fluid, and latex skin [39]. Additional physical parameters such as force and frequency were measured by Wettels et al. using an array of integrated wires within a biomimetic model of the human digit [9]. Our MEMS-based approach, featuring an integrated stiction valve, reduces the sensor footprint drastically $(250-500 \mu \mathrm{m})$, utilizes only two electrodes, and is batch fabricated on a flexible substrate. The reduction in chamber size enables precisely patterned structures for single-point pixelated contact sensing over a defined

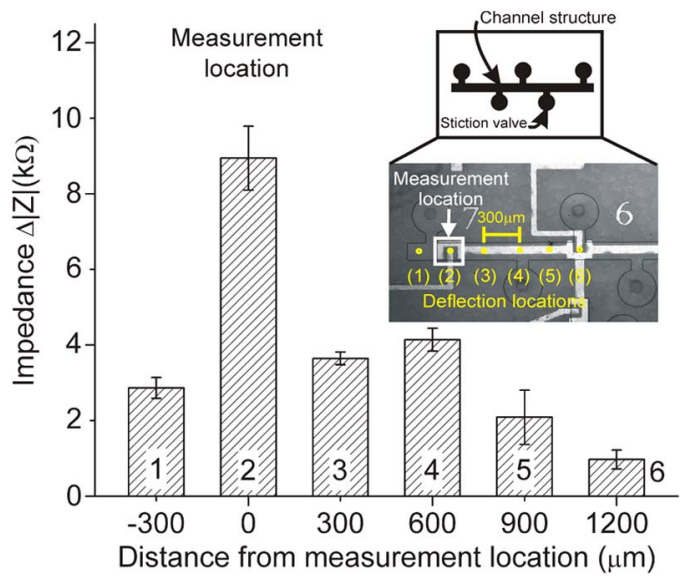

Fig. 14. Impedance response at various locations along the length of the channel (mean $\pm \mathrm{SE}, n \geq 3$ ). Inset shows the schematic representation of channel layout with peripheral stiction valves and corresponding optical micrograph with measurement and indentation sites indicated.

and controlled surface. Furthermore, the problem is reduced to the measurement of a single resistive signal correlated in a straightforward fashion to the deflection of the membrane. Although application dependent, chamber sizes on this scale can eliminate the problem of multisite contact discrimination by simply being smaller than the contacting body.

The basic requirements of an electrolyte in contact with electrodes enable applications at various size scales. EI-based sensing at submicrometer dimensions appears feasible provided that fluid handling at this scale can be realized. This could lead to applications, for example, in subcellular mechanical characterization. Unique geometric sensor configurations are also possible, extending beyond simple diaphragms to interconnected microfluidic structures.

\section{AdDitional ApplicAtions}

\section{A. Mechanotransduction Along Channels}

Additional applications of EC-MEMS can be realized by leveraging the unique liquid-based transduction mechanism. Fluidically coupled structures of varying geometries can be fabricated, such as interconnected channels, to form unique sensor configurations not possible with traditional transduction approaches. A long microfluidic channel $(150 \mu \mathrm{m} \times 2.5 \mathrm{~mm} \times$ $10 \mu \mathrm{m})$ was fabricated using the same fabrication process described previously. Stiction valves were placed peripherally along the edges of the channel for liquid encapsulation purposes. A single reference electrode spanned the entire length of the channel, while additional electrodes were placed at junctions in close proximity to the reference at specific locations within the channel. Each electrode junction acted as a single sensor element.

A mechanical stimulus (maximum deflection) was applied at various locations along the length of the channel, while the impedance response was monitored at a single junction. Deflections were detectable up to $1.2 \mathrm{~mm}$ away from the measurement site (Fig. 14). The amplitude of the measured impedance change decreased with distance. The ability to detect 


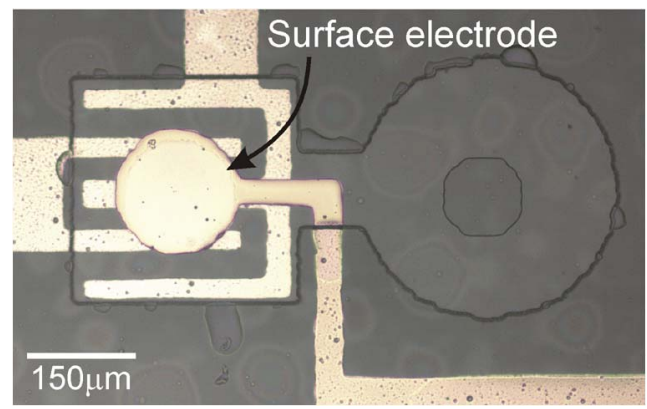

Fig. 15. Same transducer structure with an additional surface microelectrode patterned on the top exposed surface of the chamber membrane.

tactile information along the length of a channel extends the physical sensing range from that of a single point using a single chamber-based sensor to a linear range within the fluidically coupled structure. Multiple electrodes can populate a channel segment, enabling further downstream tactile detection. The mechanical transduction phenomenon along the length of the single channel could be considered biomimetic and analogous to that of neuronal signal propagation along the length of an axon. This kind of biomimetic sensing modality opens the possibilities for new bio-inspired mechanotransduction devices and applications. For example, these devices could be arranged in concentric circular patterns and applied to grasp and tactile feedback systems for minimally invasive robotic surgery, providing the ability to dynamically adjust tactile receptive fields and resolution. Alternatively, the addition of surface features such as bumps or "whiskers" could enable detection of physical parameters such as flow detection and roughness.

\section{B. Electrolysis-Based Microactuation}

In addition to sensing capabilities, EC-MEMS devices are capable of providing actuation through electrolysis of water. For demonstrative purposes, a surface microelectrode was integrated on the surface of the chamber structure. The inclusion of an electrode on the surface of the chamber enables a new device application: out-of-plane electrode actuation (Fig. 15). This configuration could be applied to neural stimulation or recording where out-of-plane microactuation of electrodes could provide improved electromechanical coupling through minute interfacial mechanical adjustments. Fabrication consists of an additional dual-layer liftoff process with a globally exposed bottom layer. This was essential to achieve continuity between metal on the substrate level and on top of the chamber. FEM of chamber deformation was conducted to establish areas of least stress for purposes of metal trace routing (data not shown). Minimum stress occurred in the connecting region between the chamber and valve. Routing metal traces through this region minimized tethering effects on the chamber by the metal during deformation and also minimized strain on the fragile traces. Platinum metallization and patterning were carried out as described previously.

The application of a constant current $(\sim 5 \mu \mathrm{A}$ for a few seconds) across the internal chamber electrodes initiated elec-
TABLE II

MAXimum Actuated Deflection For SQuARE AND CiRCUlar PARYLENE CHAMBERS $(h=10 \mu \mathrm{m}$ AND $t=2 \mu \mathrm{m})(n \geq 5)$

\begin{tabular}{c|c|c|c|c}
\hline Chamber & \multicolumn{2}{|c|}{ Circle } & \multicolumn{2}{c}{ Square } \\
\cline { 2 - 5 } size $(\mu \mathrm{m})$ & $\begin{array}{c}\text { Average } \\
(\mu \mathrm{m})\end{array}$ & $\mathrm{SE}$ & $\begin{array}{c}\text { Average } \\
(\mu \mathrm{m})\end{array}$ & $\mathrm{SE}$ \\
\hline 250 & - & - & 4.17 & 0.93 \\
\hline 300 & 4 & 0.58 & 4.2 & 0.3 \\
\hline 350 & 5.3 & 0.68 & 4.25 & 0.54 \\
\hline 400 & 5.29 & 0.65 & 4.79 & 0.61 \\
\hline 500 & 9.5 & 1.45 & - & - \\
\hline
\end{tabular}

trolysis and the evolution of hydrogen and oxygen gas within the chamber. The accumulation of this gas generated high internal pressure, causing the chamber membrane and electrode to deflect upward. Maximum deflection measurements of square and circular devices are summarized in Table II. The results indicate that larger maximum deflections were achievable as chamber dimensions increased due to increased membrane compliance with size. In general, circular chambers exhibited larger more uniform deflections than the square chambers. Electrolytic gas generation was concentrated in the center of the circular chambers, while gas generation was widespread and erratic in the square chambers. This difference is likely due to the interdigitated electrode geometry of the square chambers as well as the propensity for gases to accumulate near the edges and corners of the square chamber. The maximum achievable actuation of this device configuration was limited by the cracking pressure of the in-line stiction valve. Above this pressure threshold, the valve plate popped open, thereby limiting the amount of pressure that can be generated for actuation purposes. This pressure was estimated analytically to be about $6895 \mathrm{~Pa}(\sim 1 \mathrm{psi})$ by equating the pressure for maximum measured center deflection with valve cracking pressure. This limitation could potentially be resolved by using a multilayered configuration, thereby integrating the valve inside the chamber in such a manner that the generation of internal pressure serves to push down the valve plate, further sealing it. This would allow the buildup of high pressures and enable greater membrane deflection. Pressure generation of several hundred psi is possible by electrolysis but unlikely to be sustained within the transducer structure due to the known weak interfacial adhesion of the Parylene-Parylene interface. Strategies such as annealing, anchoring, or molten Parylene adhesion [40] may be employed to improve adhesion performance; however, a systematic comparison of the improved interfacial adhesion afforded by these techniques is still required.

\section{CONCLUSion}

EC-MEMS devices utilizing encapsulated fluid to mediate transduction and Parylene as the structural material have been designed, fabricated, and tested. Parylene microstructures, including square/circular chambers and long channels, have been evaluated and validated as highly sensitive physical transducers with applications to contact sensing, biomimetic mechanotransduction, and out-of-plane microelectrode actuation. 
These transducers are ideally suited for applications requiring highly sensitive contact force measurements of soft nonplanar surfaces in wet environments. Integration of these sensors with state-of-the-art Parylene-based retinal prosthesis will allow interrogation of important physical phenomena, in situ, for the first time. Validation experiments utilizing $e x$ vivo soft tissues are currently under way.

\section{ACKNOWLEDGMENT}

The authors would like to thank B. Kim, M. Pahwa, C. McCarty, and M. Nguyen for their invaluable assistance with device characterization. The authors would also like to thank Dr. D. Zhu and the members of the Biomedical Microsystems Laboratory, University of Southern California, for their assistance.

\section{REFERENCES}

[1] M. A. Schwarz and P. C. Hauser, "Recent developments in detection methods for microfabricated analytical devices," Lab Chip, vol. 1, no. 1, pp. 1-6, Sep. 2001.

[2] D. J. Beebe, G. A. Mensing, and G. M. Walker, "Physics and applications of microfluidics in biology," Annu. Rev. Biomed. Eng., vol. 4, no. 1, pp. 261-286, 2002.

[3] R. P. Baldwin, T. J. Roussel, M. M. Crain, V. Bathlagunda, D. J. Jackson, J. Gullapalli, J. A. Conklin, R. Pai, J. F. Naber, K. M. Walsh, and R. S. Keynton, "Fully integrated on-chip electrochemical detection for capillary electrophoresis in a microfabricated device," Anal. Chem., vol. 74, no. 15, pp. 3690-3697, Aug. 2002.

[4] W. R. Vandaveer, IV, S. A. Pasas-Farmer, D. J. Fischer, C. N. Frankenfeld, and S. M. Lunte, "Recent developments in electrochemical detection for microchip capillary electrophoresis," Electrophoresis, vol. 25, no. 21/22, pp. 3528-3549, Nov. 2004.

[5] D. Erickson and D. Li, "Integrated microfluidic devices," Anal. Chim. Acta, vol. 507, no. 1, pp. 11-26, Apr. 2004.

[6] S. Z. Hua, F. Sachs, D. X. Yang, and H. D. Chopra, "Microfluidic actuation using electrochemically generated bubbles," Anal. Chem., vol. 74, no. 24, pp. 6392-6396, Dec. 2002.

[7] A. P. Papavasiliou, D. Liepmann, and A. P. Pisano, "Electrolysis-bubble actuated gate valve," in Proc. Solid-State Sensor Actuator Workshop, Hilton Head, SC, 2000, pp. 48-51.

[8] W. H. Coulter, "Means for counting particles suspended in a fluid," U.S. Patent 2656508 , Oct. 20, 1953

[9] N. Wettels, V. J. Santos, R. S. Johansson, and G. E. Loeb, "Biomimetic tactile sensor array," Adv. Robot., vol. 22, no. 8, pp. 829-849, 2008.

[10] A. R. Russel, "A tactile sensory skin for measuring surface contours," in Proc. IEEE Region 10 Int. Conf.-Technology Enabling Tomorrow: Computers, Communications and Automation Towards the 21st Century, Melbourne, Australia, 1992, pp. 262-266.

[11] A. R. Russel and S. Parkinson, "Sensing surface shape by touch," in Proc. IEEE Int. Conf. Robot. Autom., Atlanta, GA, 1993, pp. 423-428.

[12] D. A. Ateya, A. A. Shah, and S. Z. Hua, "Impedance-based response of an electrolytic gas bubble to pressure in microfluidic channels," Sens. Actuators A, Phys., vol. 122, no. 2, pp. 235-241, Aug. 2005.

[13] J. D. Weiland, W. Liu, and M. S. Humayun, "Retinal prosthesis," Апnи. Rev. Biomed. Eng., vol. 7, pp. 361-401, 2005.

[14] D. C. Rodger, A. J. Fong, W. Li, H. Ameri, A. K. Ahuja, C. Gutierrez, I. Lavrov, H. Zhong, P. R. Menon, E. Meng, J. W. Burdick, R. R. Roy, V. R. Edgerton, J. D. Weiland, M. S. Humayun, and Y.-C. Tai, "Flexible parylene-based multielectrode array technology for high-density neural stimulation and recording," Sens. Actuators B, Chem., vol. 132, no. 2, pp. 449-460, Jun. 2008.

[15] L. Colodetti, J. D. Weiland, S. Colodetti, A. Ray, M. J. Seiler, D. R. Hinton, and M. S. Humayun, "Pathology of damaging electrical stimulation in the retina," Exp. Eye Res., vol. 85, no. 1, pp. 23-33, Jul. 2007.

[16] D. Reigada, W. Lu, M. Zhang, and C. H. Mitchell, "Elevated pressure triggers a physiological release of ATP from the retina: Possible role for pannexin hemichannels," Neuroscience, vol. 157, no. 2, pp. 396-404, Nov. 2008.

[17] A. B. Majji, M. S. Humayun, J. D. Weiland, S. Suzuki, S. A. D’Anna, and E. de Juan, Jr., "Long-term histological and electrophysiological results of an inactive epiretinal electrode array implantation in dogs," Invest. Ophthalmol. Vis. Sci., vol. 40, no. 9, pp. 2073-2081, 1999.

[18] B. C. Basinger, A. P. Rowley, K. Chen, M. S. Humayun, and J. D. Weiland, "Finite element modeling of retinal prosthesis mechanics," J. Neural Eng., vol. 6, no. 5, p. 055 006, Oct. 2009.

[19] Y.-B. Lu, K. Franze, G. Seifert, C. Steinha, F. Kirchhoff, H. Wolburg, J. Guck, P. Janmey, E.-Q. Wei, J. Kas, and A. Reichenbach, "Viscoelastic properties of individual glial cells and neurons in the CNS," PNAS, vol. 103, no. 47, pp. 17 759-17 764, Nov. 2006.

[20] H. A. Quigley, "Neuronal death in glaucoma," Prog. Retin. Eye Res., vol. 18, no. 1, pp. 39-57, Jan. 1999.

[21] I. Suzuma, T. Murakami, K. Suzuma, H. Kaneto, D. Watanabe, T. Ojima, Y. Honda, H. Takagi, and N. Yoshimura, "Cyclic stretch-induced reactive oxygen species generation enhances apoptosis in retinal pericytes through c-Jun $\mathrm{NH}_{2}$-terminal kinase activation," Hypertension, vol. 49, no. 2, pp. 347-354, Feb. 2007.

[22] C. A. Gutierrez and E. Meng, "A dual function parylene-based biomimetic tactile sensor and actuator for next generation mechanically responsive microelectrode arrays," in Proc. Transducers, Denver, CO, 2009, pp. 2194-2197.

[23] H. Zhang, S. Wang, and Y. Xu, "Study and applications of a parylene self-sealing structure," in Proc. MEMS, 2006, pp. 282-285.

[24] L. S. Robblee and T. L. Rose, "The electrochemistry of electrical stimulation," in Proc. Annu. Int. Conf. IEEE Eng. Med. Biol. Soc., Philadelphia, PA, 1990, pp. 1479-1480.

[25] J. E. B. Randles, "Kinetics of rapid electrode reactions," in Proc. Discuss. Faraday Soc., 1947, pp. 11-19.

[26] L. S. Robblee and T. L. Rose, "Electrochemical guidelines for selection of protocols and electrode materials for neural stimulation," in Neural Prostheses Fundamental Studies, W. F. Agnew and D. B. Mcreery, Eds. Englewood Cliffs, NJ: Prentice-Hall, 1990, pp. 26-59.

[27] L. Wolgemuth, "Assessing the performance and suitability of parylene coating," Med. Device Diagn. Ind., vol. 22, no. 8, pp. 42-49, 2000.

[28] Cookson Specialty Coating Systems. [Online]. Available: http://www. scscoatings.com/parylene_knowledge/specifications

[29] C. A. Gutierrez and E. Meng, "Improved self-sealing liquid encapsulation in parylene structures by integrated stackable annular-plate stiction valve," in Proc. IEEE MEMS, Hong Kong, 2010, pp. 524-527.

[30] C. H. Mastrangelo and C. H. Hsu, "Mechanical stability and adhesion of microstructures under capillary forces. I. Basic theory," J. Microelectromech. Syst., vol. 2, no. 1, pp. 33-43, Mar. 1993.

[31] C. H. Mastrangelo and C. H. Hsu, "Mechanical stability and adhesion of microstructures under capillary forces. II. Experiments," J. Microelectromech. Syst., vol. 2, no. 1, pp. 44-55, Mar. 1993.

[32] Z. Wang and Y. Xu, "Theoretical and experimental study of annularplate self-sealing structures," J. Microelectromech. Syst., vol. 17, no. 1, pp. 185-192, Feb. 2008.

[33] S. Matsumoto and N. Ichikawa, "New methods for liquid encapsulation in polymer MEMS structures," in Proc. MEMS, Tucson, AZ, 2008, pp. $415-418$.

[34] A. Tanioka, N. Fukushima, K. Hasegawa, K. Miyasaka, and N. Takahashi, "Permeation of gases across the poly(chloro-p-xylylene) membrane," $J$. Appl. Polym. Sci., vol. 54, no. 2, pp. 219-229, Oct. 1994.

[35] P. R. Menon, W. Li, A. Tooker, and Y. C. Tai, "Characterization of water vapor permeation through thin film parylene C," in Proc. Transducers, Denver, CO, 2009, pp. 1892-1895.

[36] M. D. Giovanni, Flat and Corrugated Diaphragm Design Handbook, vol. 11. New York: Marcel Dekker, 1982.

[37] K. Kasten, N. Kordas, H. Kappert, and W. Mokwa, "Capacitive pressure sensor with monolithically integrated CMOS readout circuit for high temperature applications," Sens. Actuators A, Phys., vol. 97/98, pp. 8387, Apr. 2002.

[38] P. Hille, R. Höhler, and H. Strack, "A linearisation and compensation method for integrated sensors," Sens. Actuators A, Phys., vol. 44, no. 2, pp. 95-102, Aug. 1994.

[39] M. Helsel, J. N. Zemel, and V. Dominko, "An impedance tomographic tactile sensor," Sens. Actuators, vol. 14, no. 1, pp. 93-98, 1988.

[40] R. Huang and Y. C. Tai, "Parylene to silicon adhesion enhancement," in Proc. Transducers, Denver, CO, 2009, pp. 1027-1030. 


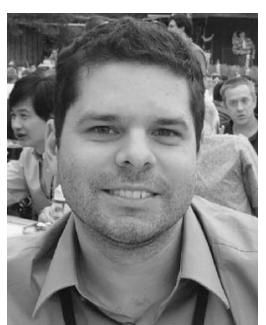

Christian A. Gutierrez (M'09) received B.S. degrees in electrical engineering and business economics and management and the M.S. degree in electrical engineering from the California Institute of Technology (Caltech), Pasadena, in 2005 and 2006, respectively. He is currently working toward the $\mathrm{Ph} . \mathrm{D}$. degree in biomedical engineering (under fellowship) at the University of Southern California (USC), Los Angeles.

$\mathrm{He}$ is also currently with the National Science Foundation's Biomimetic Microelectronic Systems Engineering Research Center, USC, where he is a Copresident of the student leadership council. His past research was carried out in the Caltech Micromachining Laboratory and was focused on identifying, analyzing, and implementing power harvesting and storage circuitry for microelectromechanical-systems-based power harvesting devices. He is currently conducting research toward the development of novel micro- and nanotechnologies for biomedical applications. His specific research is focused on the development of retinal prosthesis technologies to restore sight to the blind.

Mr. Gutierrez was a recipient of the Caltech Presidential Scholarship and is currently a Bill Gates Millennium Scholar. He was also a recipient of the USC Viterbi School of Engineering Kunzel Fellowship.

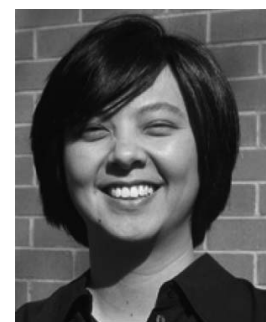

Ellis Meng (M'02-SM'09) received the B.S. degree in engineering and applied science and the M.S. and Ph.D. degrees in electrical engineering from the California Institute of Technology (Caltech), Pasadena, in 1997, 1998, and 2003, respectively.

Since 2004, she has been with the Department of Biomedical Engineering, University of Southern California (USC), Los Angeles, where she is currently an Associate Professor. She currently holds a joint appointment with the Ming Hsieh Department of Electrical Engineering, USC. She is currently the Thrust Leader for Interface Technology and the Associate Director of Education and Student Diversity Researcher at the National Science Foundation (NSF)'s Biomimetic Microelectronic Systems Engineering Research Center, USC. She held the Viterbi Early Career Chair in the USC Viterbi School of Engineering. Her research interests include biological microelectromechanical systems, implantable biomedical microdevices, microfluidics, multimodality integrated microsystems, and packaging.

Dr. Meng is a member of Tau Beta Pi, the Biomedical Engineering Society, the Society of Women Engineers, and the American Society for Engineering Education. She was a recipient of the Intel Women in Science and Engineering Scholarship, the Caltech Alumni Association Donald S. Clark Award, and the Caltech Special Institute Fellowship. She has also received the NSF CAREER and Wallace H. Coulter Foundation Early Career Translational Research Awards. 